\title{
The Value of Positron Emission Tomography in the Mediastinal Lymph Node Metastasis of the Patients Operated for Non-Small Cell Lung Cancer
}

\author{
Fazli Yanik ${ }^{1 *}$, Yekta Altemur Karamustafaoglu ${ }^{1}$, Ali Sarıkaya ${ }^{2}$ and Yener Yoruk ${ }^{1}$ \\ ${ }^{1}$ Deparment of Thoracic Surgery, Trakya University, Turkey
}

${ }^{2}$ Deparment of Nuclear Medicine, Trakya University, Turkey

Submission: April 04, 2018; Published: May 09, 2018

*Corresponding author: Fazli Yanik, Deparment Thoracic Surgery, Trakya University, Gogus Cerrahi Ad, Kat: 4, 22000, Edirne, Turkey, Tel: +902842355936; Fax: +902842355936; Email: fazliyanik@hotmail.com

\begin{abstract}
Background: In recent years, Positron Emission Tomography (PET-CT) has been used increasingly in the diagnosis of thoracic malignancies, in the evaluation of staging, distant organ metastasis, and post-chemotherapy response. The role of PET/CT in the mediastinal lymph node stage still remains controversial due to its high false positives and false negatives.

Aim: We aim to evaluate the diagnostic value and false negative and positive results of PET/CT on the mediastinal lymph node metastasis in the patients operated for Non-Small Cell Lung Cancer (NSCLC).

Material and Methods: The study included 164 patients with NSCLC who underwent mediastinoscopy or anatomic resection with mediastinal lymph node dissection between January 2008 and January 2018 in Trakya University Thoracic Surgery Department. There were 34 females, 130 male patients whose ages were between $20-88$ years (mean: 60,5). We retrospectively compared the mediastinal lymph node involvement preoperatively PET-CT findings and histopathologic results in patients with NSCLC who were not received previously chemotherapy or radiotherapy. Metabolic characterizations of mediastinal lymph nodes and SUVmax values were recorded.
\end{abstract}

Results: A total of 682 lymph node samples were performed in this study. The median five lymph nodes (4-13 lymph nodes) dissection was performed per average case. The mean SUVmax value of the True Negative, True Positive, False Negative and False Positive cases was 0,29(0-1,8); 2,96(1,8-6,4); $0(0) ; 3,4(1,9-6,8)$, respectively. Also sensitivity, specificity, positive predictive value, and negative predictive value for mediastinal lymph nodes were found $77,7 \%, 93,5 \%, 38,8 \%$ and $92,9 \%$, respectively.

Conclusion: We conclude that, invasive mediastinal staging with video mediastinoscopy (VAM) should be performed in NSCLC due to low sensitivity and positive predictive value of PET CT.

Keywords: Thoracic Surgery; PET/CT; Lymph node; Non-Small Cell Lung Tumor

Abbreviations: TN: True Negative; TP: True Positive; FN: False Negative; FP: False Positive; PET-CT: Positron Emission Tomography; CT: Computerized Tomography; NSCLC: Non-Small Cell Lung Cancer; EBUS: Endobronchial Ultrasonography

\section{Introduction}

In recent years, Positron Emission Tomography (PET-CT) has been used increasingly in the diagnosis of thoracic malignancies, in the evaluation of staging, distant organ metastasis, and postchemotherapy response [1,2]. PET-CT is a new imaging format that provides metabolic and anatomical information about the tumor. Non-invasive imaging using tomographic images and quantitative parameters that reflect the perfusion, metabolic activity and vitality of the tissues [3]. Since PET-CT images have been anatomic localization and spatial resolution insufficiency, the anatomical localization of the lesions can be performed frequently and the sections are combined using Computerized Tomography (CT) and this is called PET-CT. False positivite lesions are with high 18-fluorodeoxyglucose (F18-FDG) uptake when not malignant and false negativites are low-F18-FDG uptake lesions when they are malignant. The role of PET / CT in the mediastinal lymph node stage still remains controversial due to its high false positives and false negatives. Invasive staging methods continue to be the gold standard in the stage of the metastatic lymph node [4]. We aim to evaluate the diagnostic value and false negative-false positive results of PET/CT on the mediastinal lymph node metastasis in the patients who underwent mediastinoscopy or anatomic resection for NonSmall Cell Lung Cancer (NSCLC).

\section{Material and Methods}

In this study; we analyzed the mediastinal lymph node involvement preoperatively PET-CT findings and histopathologic 
results in patients with NSCLC who were not received previously chemotherapy or radiotherapy. NSCLC was diagnosed in all patients by bronchoscopy, fine needle aspiration biopsy or intraoperative frozen sections. The study included patients with NSCLC who underwent mediastinoscopy or anatomic resection with mediastinal lymph node dissection. Patients under 18 years of age, given chemo-radiotherapy chemo-radiotherapy, non-NSCLC's, uncontrolled diabetics, N1 positive tumors were excluded from the study. The cases were evaluated according to age, sex, tumor type, Maximum standart uptake value (SUVmax) values of mediastinal lymph nodes, pathology and PET/CT imaging results. Oral intake was stopped 6-8 hours before the shots, and blood glucose level were kept below $150 \mathrm{mg} / \mathrm{dl}$ in all cases. After blood glucose level and blood pressure was measured, 18 F FDG dose $(8-15 \mathrm{mCi} / \mathrm{kg})$ calculated according to body weight was administered intravenously. After injection, the patients were taken to the waiting room about 45-60 minutes. The PET/CT device (G.E. Discovery STE8), a combination of a tomography unit with a cross-sectional thickness of five $\mathrm{mm}$ and a PET unit containing a Bismuth Germenate block detector, was used for the thorax images of the cases. Images were taken from the upper part of the vertex to the middle of the thigh. PET/CT results were assessed by nuclear medicine specialists.

We analyzed retrospectively 164 patients who operated for NSCLC and who had PET-CT imaging between January 2008 and January 2018 in Trakya University Thoracic Surgery Department. Metabolic characterization of mediastinal lymph nodes and SUVmax values were recorded. There were 34 females, 130 male patients whose ages were between 20-88 years (mean: 60,5). For every patient, SUV max values were calculated and sizes of the lesions and lymph nodes were measured. Finally, PET-CT results were compared with histopathologic data. It was aimed to evaluate the involvement of PET-CT in the mediastinal lymph nodes together with the pathologic outcome, to determine false negativity and false positives. The mean SUVmax values of mediastinal lymph nodes were calculated according to pathology results. All tumors with malignant mediastinal lymph node involvement in PET-CT, especially central located or over 3 $\mathrm{cm}$ in diameter tumors were first performed VAM. Patients with mediastinal lymph node metastasis detected by VAM were sent to oncologic treatment. An anatomic resection and a mediastinal lymph node dissection were performed in the patients who were not detected with metastasis by VAM or peripherally located squamous tumors less than $3 \mathrm{~cm}$ in diameter without VAM. Complete thoracic lymphadenectomy was routinely performed; it consisted of enbloc resection of all lymph nodes that were accessible in the mediastinum and hilum of lung. Intraoperative N2 was not detected.

\section{Data Analysis}

A statistical analysis was performed using the Statically Package for the Social Science program (SPSS, 20.0) Demographics, pathological data and PET/CT results are expressed in mean values. Frequencies and percentages were used for the categorical measures. Sensitivity, selectivity, positive predictive value, negative predictive value and accuracy criteria were used for comparison of the different diagnostic methods.

\section{Results}

Table 1: True negative, true positive, false negative, false positive results in mediastinal lymph nodes of PET / CT.

\begin{tabular}{|c|c|c|}
\hline \multicolumn{3}{|c|}{ False Positive Cases } \\
\hline $\begin{array}{l}\text { Pathological } \\
\text { Diagnosis }\end{array}$ & $\mathrm{n}(11)$ & SUVmax(mean): 3,4 \\
\hline Reactive Hyperplasia & 4 & $2,4(2-2,8)$ \\
\hline Anthracosis & 3 & $2,3(1,9-2,9)$ \\
\hline Sarcoidosis & 2 & $6(5,2-6,8)$ \\
\hline Tuberculosis & 2 & $2,9(2,1-3,7)$ \\
\hline \multicolumn{3}{|c|}{ False Negative Cases } \\
\hline $\begin{array}{l}\text { Pathological } \\
\text { Diagnoses }\end{array}$ & $\mathrm{n}(2)$ & SUVmax (mean): 0 \\
\hline Adenocarsinoma & 0 & $0(0)$ \\
\hline \multicolumn{3}{|c|}{ True Negative Cases } \\
\hline $\begin{array}{l}\text { Pathological } \\
\text { Diagnosis }\end{array}$ & n (144) & SUVmax(mean): 0,29 \\
\hline $\begin{array}{l}\text { Squamous Cell } \\
\text { Carcinoma }\end{array}$ & 65 & $0,3(0-1,8)$ \\
\hline Adenocarcinoma & 62 & $0,2(0-1,1)$ \\
\hline Large cell Carcinoma & 6 & $0(0)$ \\
\hline Carcinoid Tumor & 5 & $0(0)$ \\
\hline $\begin{array}{c}\text { Lymphepithelial like } \\
\text { Carcinoma }\end{array}$ & 2 & $0(0)$ \\
\hline $\begin{array}{c}\text { Sarcomatoid } \\
\text { Carcinoma }\end{array}$ & 2 & $0,75(0-1,5)$ \\
\hline $\begin{array}{l}\text { Adenoskuamoz } \\
\text { Karsinom }\end{array}$ & 2 & $0,8(0-1,6)$ \\
\hline \multicolumn{3}{|c|}{ True Positive Cases } \\
\hline $\begin{array}{l}\text { Pathological } \\
\text { diagnosis }\end{array}$ & n (7) & $\operatorname{SUVmax}($ mean $)(2,96)$ \\
\hline $\begin{array}{l}\text { Squamous Cell } \\
\text { Carcinoma }\end{array}$ & 3 & $3,7(2,8-6,4)$ \\
\hline Adenocarcinoma & 2 & $3,2(3-3,4)$ \\
\hline Large Cell Carsinoma & 2 & $2(1,8-2,2)$ \\
\hline
\end{tabular}

${ }^{*}$ False positive $(F P)=11$ cases SUVmax $\left.=3,4(1,9-6,8)\right)$; False negative $(F N)=2$ cases SUVmax 0

*True Positive (TP) = 7 cases SUVmax $=2,96(1,8-6,4)$; True negative $(\mathrm{TN})=144$ cases SUVmax 0,29 $(0-1,8)$

\section{SUVmax: Maximum Standardized Uptake Value}

In 146 cases mediastinal lymph node involvement was not detected, whereas in 18 cases, mediastinal lymph node involvement was detected in favor of malignancy in PET/CT. Four of this 18 case first performed the endobronchialultrasonography (EBUS) fine needle biopsy, but none were diagnosed. VAM was performed 72 patients included these also 18 patients. True positive was 7 patients: Four patients (upper paratracheal n:2, 
lower paratracheal $n: 1$, subcarinal $n: 1$ ) with $\mathrm{N} 2$ detected in VAM and these patients received chemotherapy. N2 lymph node positivity was detected in three cases (subcarinal $\mathrm{n}: 2$, paraaortic $\mathrm{n}: 1)$ after postoperative pathological examination. These cases were sent to adjuvant chemotherapy. Other 11 of 18 cases was false positive cases, surgical resection continued in these cases (Table 1).

Subcarinal lymph node was detected tumor positive in two of 146 PET / CT metastases negative mediastinal lymph node by mediastinoscopy and thoracotomy and patients recieved chemotherapy (Table 1). Lymph node negative patients were operated. After the histopathologic examination, 35 patients
$(21,3 \%)$ were in stage IA, $54(32,9 \%)$ patients in stage IB, $43(26,2 \%)$ patients in stage IIA, $23(14 \%)$ patients in stage IIB, $9(5,4 \%)$ patients in stage IIIA. 160 patients underwent surgery. Lobectomy for 114 (22 of them was VATS lobectomy), pneumonectomy for 27 , bilobectomy for 11 , and segmentectomy for 8 (2 of them was VATS segmentectomy) were performed. Tumor histopathological types were squamous cell carcinoma in 73 patients, adenocarcinoma in 68 patients, other 19(large cell $\mathrm{n}: 8,5$ carcinoid $\mathrm{n}: 5$, lymphoepithelioma-like carcinoma $\mathrm{n}: 2$, sarcomatoid type lung carcinoma $\mathrm{n}: 2$, adenosquamous carcinoma n:2). Median SUVmax values of malignant / benign tumors of mediastinal lymph nodes can be seen in Figure 1.

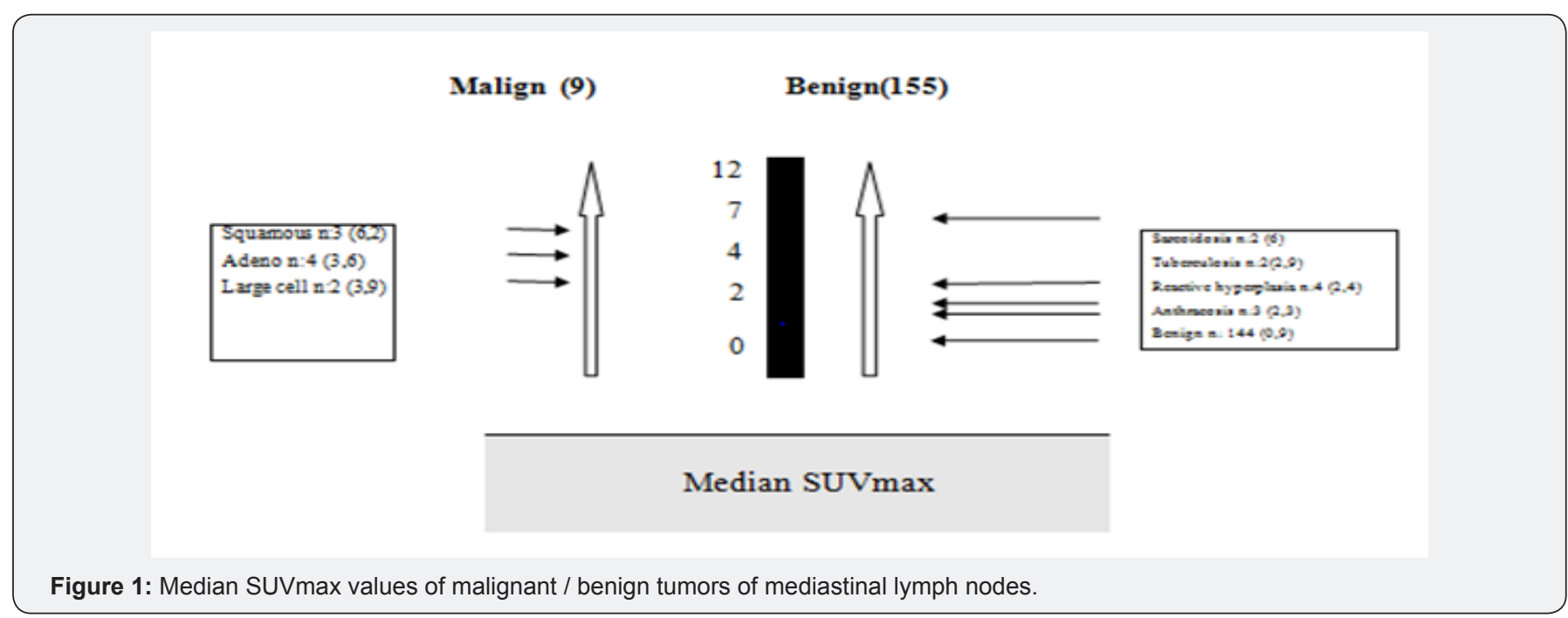

Table 2: Measures of diagnostic accuracy positron emission tomography for meditational lymph nodes.

\begin{tabular}{|c|c|c|}
\hline & Pathology (+) Cases(9) & Pathology (-) Cases (155) \\
\hline PET/CT $(+)$ cases $(18)$ & $\mathrm{TP}=7(\mathrm{~A})$ & $\mathrm{FP}=11(\mathrm{~B})$ \\
\hline PET/CT(-) cases (146) & $\mathrm{FN}=2(\mathrm{C})$ & $\mathrm{TN}=144(\mathrm{D})$ \\
\hline \multicolumn{3}{|c|}{ Number of True Positive(TP) cases: 7} \\
\hline \multicolumn{3}{|c|}{ Number of True Negative (TN) cases: 144} \\
\hline \multicolumn{3}{|c|}{ Number of False Negative (FN) cases: 2} \\
\hline \multicolumn{3}{|c|}{ Number of False Positive (FP) cases: 11} \\
\hline \multicolumn{3}{|c|}{ Sensitivity (Correct positivity rate):A/A+C=77,7\% } \\
\hline \multicolumn{3}{|c|}{ Specificity (True negativity rate):D/B+D=93,5\% } \\
\hline \multicolumn{3}{|c|}{ Positive Predictive Value (PPV): A/A+B=38,8\% } \\
\hline \multicolumn{3}{|c|}{ Negative Predictive Value (NPV):D/C+D=92,9\% } \\
\hline \multicolumn{3}{|c|}{ Accuracy $=(\mathrm{TP}+\mathrm{TN}) /(\mathrm{TP}+\mathrm{FP}+\mathrm{TN}+\mathrm{FN})=98,6 \%$} \\
\hline \multicolumn{3}{|c|}{ False Negativity Rate: $\mathrm{FNR}=(1-$ Sensitivity $)=\mathrm{C} /(\mathrm{A}+\mathrm{C})=\mathrm{FN} /(\mathrm{FN}+\mathrm{TP})=22,3 \%$} \\
\hline \multicolumn{3}{|c|}{ False Positivity Rate: FPR $=(1$ - Specificity $)=B /(B+D)=F P /(F P+T N)=6,5 \%$} \\
\hline
\end{tabular}

PET/CT: Positron Emission Tomography / Computed Tomography; TN: True Negative; TP: True Positive; FN: False Negative; FP: False Positive

A total of 682 lymph node samples were removed in this study. The median five lymph nodes (4-13 lymph nodes) dissection was performed per average case. True Negative (TN), True Positive (TP), False Negative (FN), False Positive (FP) results in mediastinal lymph nodes of PET / CT was summarized in Table 1. The mean SUVmax value of the TN, TP, FN and FP cases were $0,29(0-1,8) ; 2,96(1,8-6,4) ; 0(0) ; 3,4(1,9-6,8)$, respectively. The TP lymph nodes were greater than $1 \mathrm{~cm}$ in diameter and 
two patients'with FN of lymph nodes was smaller than $1 \mathrm{~cm}$ in diameter. Sensitivity, specificity, positive predictive value, and negative predictive value for mediastinal lymph nodes were found $77,7 \%, 93,5 \%, 38,8 \%$ and $92,9 \%$, respectively (Table 2).

\section{Discussion}

PET/CT has been widely used in the diagnosis of thoracic malignancies, staging, determination of distant organ metastasis and evaluation of post-chemotherapy response. Treatment protocol is closely related to the stage of the disease in patients with NSCLC. For this reason, proper staging is great importance in NSCLC. The rate of mediastinal lymph node involvement at diagnosis is $28 \%-38 \%$. However PET/CT as a new imaging method in diagnosis and staging of lung cancer, many studies have reported false positives and false negatives of it. Also PET/ $\mathrm{CT}$ has a low sensitivity and positive predictive value (PPV). This can lead to many overdiagnosis or misdiagnosis in daily practice. Many studies in the literature on PET/CT mediastinal lymph node evaluation for non-small cell lung tumors has shown that sensitivity, specificity, positive and negative predictive values are higher than only CT [1-6]. F18-FDG is not a cancer specific agent; but it also may be positive uptake sarcoidosis, tuberculosis, fungal infection, and cerebral abscess. However, unlike benign pathologies, FDG uptake following malignant lesions is partially beneficial in differential diagnosis [5].

In the study conducted by Bellek et al. [7] sensitivity, specificity, PPV, NPV and accuracy for staging mediastinal lymph nodes were $86,7 \%, 65,5 \%, 56,5 \%, 90,5 \%$ and $72,7 \%$, respectively. The false-negative rate was $13,3 \%$ while the falsepositive rate was $34,5 \%$ according to their study. In our study, sensitivity was $77,7 \%$, specificity was $93,5 \%$, PPV was $38,8 \%$, NPV was $92,9 \%$ and accuracy was $98,6 \%$. Our findings are consistent with previous literature and the PPV is lower than the $\mathrm{NPV}$. As the reason for this, infectious and inflammatory diseases is responsible for false positive results.

The literature on false positive or false negative results of PET-CT is limited. Takamochi et al. [8] reported the false positive rate as $14 \%$ and false negative rate as $20 \%$. False negativity rate was $22,3 \%$ and false positivity rate was $6,5 \%$ in our study. All infections and inflammations with active macrophages, especially granulomatous diseases, are known to be caused by the potential false positives in PET/CT. The most common causes of false positives are tuberculosis, sarcoidosis, coccidomycosis, aspergillosis and some other infections. On the other hand, low metabolic activity tumors may give false negative results in PET/ CT. In addition, due to the limited spatial resolution of PET / CT, the false negative result rate is increased in lesions smaller than $1 \mathrm{~cm}$ (especially $<6 \mathrm{~mm}$ ). Hyperglycemia may also potentially lead to false negativity because competitively inhibited FDG may reduce tumor "uptake" [5-9]. While PET-CT study was evaluated; false positive and false negative findings should be considered. Muscle tissue, brown adipose tissue, various physiological involvements such as thymus hyperplasia after chemotherapy, lung or mediastinal infections or inflammations, and non-malignant tumors-related involvement lead to false positive results [9]. In our study, low sensivity and fairly low positive predictive value was detected. This attributed to 11 cases detected as false positive due to reactive hyperplasia, anthracosis and granulomatous inflammation. This is due to the high incidence of infectious diseases in our country. Also false negative two cases with less than $1 \mathrm{~cm}$ tumor positive mediastinal lymphadenopathy. Because the sensitivity of PET/ CT is very low in lesions smaller than $1 \mathrm{~cm}$ [10]. Kandemir et al. [11] reported that when the SUV(max) value of 2,5 was used, the sensitivity, PPV, and diagnostic accuracy of the PET/CT were $100 \%, 65,4 \%$, and $65,4 \%$ respectively. Also they were calculated the SUVmax cut-off value with the highest diagnostic accuracy $(75 \%)$ as 6.3 . They concluded that although a SUVmax cut-off value of 6.3 increases specificity and diagnostic accuracy, the value of 2,5 and above can give more optimal results.

In the meta-analysis de Langen et al. [12] reported that the lymph nodes measured smaller than $1 \mathrm{~cm}$ without FDG uptake, malignancy was detected in less than $5 \%$ of them. Therefore there was no need for further mediastinal examination in these cases. According to the European Society of Thoracic Surgeons, in case of computed tomography enlarged than $1 \mathrm{~cm}$ or PET/ CT positive mediastinal lymph nodes, tissue confirmation is indicated. First endobronchial ultrasonography (EBUS) or esophageal ultrasonography (EUS) should be tried, if negative mediastinoscopy should be performed. When there are no enlarged lymph nodes than $1 \mathrm{~cm}$ in $\mathrm{CT}$ and when there is no uptake in lymph nodes on PET-CT, direct surgical resection with systematic lymph node dissection is indicated for periferical located and $\leq 3 \mathrm{~cm}$ tumours [13]. In our study VAM was performed in cases with lymph node larger than $1 \mathrm{~cm}$ and PET/ CT positive. EBUS/EUS was performed only PET/CT positive four patients but none were diagnosed. When there are no enlarged lymph nodes than $1 \mathrm{~cm}$ in short axis on $\mathrm{CT}$ and when there is no uptake in lymph nodes on PET-CT, direct surgical resection with systematic nodal dissection is performed for tumors $\leq 3 \mathrm{~cm}$ and peripheral except central tumors or N1 nodes, adenocarcinoma, tumors $3 \mathrm{~cm}>$ and FDG positive lymph nodes.

\section{Conclusion}

We conclude that; evaluation of mediastinal lymph nodes by PET-CT alone is not appropriate for the stage of non-small cell lung cancer. The granulomatous-infectional diseases may lead to false positive results. In the mediastinal lymph node stage of NSCLC, for PET-CT positive lesions, the choice between endoscopic staging with EBUS/EUS and transbronchial biopsy or VAM depends on experience to minimal requirements for staging.

\section{References}

1. Schmidt Hansen M, Baldwin DR, Hasler E, Zamora J, Abraira V, et al. (2014) PET-CT for assessing mediastinal lymph node involvement in patients with suspected resectable non-small cell lung cancer. Cochrane Database Syst Rev 13(11): 1-167. 
2. JungMinCh, HyunJu L, JinMoG, Ho Young L, Jong JL, et al. (2006) False Positive and False Negative FDG-PET Scans in Various Thoracic Diseases. Korean J Radiol 7(1): 57-69.

3. Kostakoglu L, Agress H, Goldsmith SJ (2003) Clinical role of FDG PET in evaluation of cancerpatients. RadioGraphics 23: 315-340.

4. Chang J M, Lee H J, Goo J M, Lee H Y (2006) False Positive and False Negative FDG-PET Scans in Various Thoracic Diseases. KJR 7(1): 57-69.

5. Higashi T, Saga T, Nakamoto Y, Ishimori T, Mamede MH, et al. (2002) Relationship between retention index in dual-phase 18F-FDG PET, and hexokinase-II and glucose transporter-1 expression in pancreatic cancer. J Nucl Med 43(2): 173-180.

6. Frisher Ravens A, Bahuslavizki KH, Brandt Lars (2003) Mediastinal lymp node involvement in potential resectable lung cancer: comparison of CT, positron emission tomography, and endoscopic ultrasonography with and without fine-needle aspiration. Chest 123: 442-451.

7. Bellek E, Erturan SS, Hallac M, Sonmezoglu K, Kaynak K, et al. (2010) The Role of PET-CT in Mediastinal Lymph Node Staging in Non-small Cell Lung Cancer. Eurasian J Pulmonol 12(1): 13-20.

8. Takamochi K, Yoshida J, Murakami K, Niho S, Ishii G, et al. (2005) Pitfalls in lymph node staging with positron emission tomography in non-small cell lung cancer patients. Lung Cancer 47(2): 235-242.
9. Bryant AS, Cerfolio RJ (2006) The maximumstandardized uptake values on integrated FDG- PET/CT is useful in differentiating benign from malignant pulmonary nodules AnnThorac Surg 82(3): 10161020 .

10. De Leyn P, Vansteenkiste J, Cuypers P ve ark (1997) Role of cervical mediastinoscopy in staging of non-small cell lung cancer without enlarged mediastinal lymph nodes on CT scan. Eur J Cardiothorac Surg 12(5): 706-712.

11. Kandemir Z, Sentürk A, Ozdemir E, Yildirim N, Hasanoğlu HC, et al (2015) The evaluation of hypermetabolic mediastinal-hilar lymph nodes determined by PET/CT in pulmonary and extrapulmonary malignancies: correlation with EBUS-TBNA. Turk J Med Sci 45(6): 1234-1242.

12. de Langen AJ, Raijmakers P, Riphagen I, Paul MA, Hoekstra OS (2006) The size of mediastinal lymph nodes and its relation with metastatic involvement: a meta-analysis. Eur J Cardiothorac Surg 29(1): 26-29.

13. Leyna PD, Doomsb C, Kuzdzalc J, Lardinoisd D, Passlicke B, et al. ((2014)) Revised ESTS guidelines for preoperative mediastinal lymph node staging for non-small-cell lung cancer. European Journal of Cardio-Thoracic Surgery p. 1-12.

\section{Your next submission with Juniper Publishers} will reach you the below assets

- Quality Editorial service

- Swift Peer Review

- Reprints availability

- E-prints Service

- Manuscript Podcast for convenient understanding

- Global attainment for your research

- Manuscript accessibility in different formats

( Pdf, E-pub, Full Text, Audio)

- Unceasing customer service

Track the below URL for one-step submission https://juniperpublishers.com/online-submission.php 